\title{
A Novel Planar Slot Antenna Structure for 5G Mobile Networks Applications
}

\author{
Kamal Bouzakraoui", Ahmed Mouhsen, Abdelkader Youssefi \\ MMII Laboratory of Settat Science Faculty, Settat University Hassan I, Settat, Morocco \\ Email address: \\ bouzakraoui.kamal@gmail.com (K. Bouzakraoui), mouhsen.ahmed@gmail.com (A. Mouhsen), ab.youssefi@gmail.com (A. Youssefi) \\ ${ }^{*}$ Corresponding author
}

\section{To cite this article:}

Kamal Bouzakraoui, Ahmed Mouhsen, Abdelkader Youssefi. A Novel Planar Slot Antenna Structure for 5G Mobile Networks Applications. Journal of Electrical and Electronic Engineering. Vol. 5, No. 4, 2017, pp. 111-115. doi: 10.11648/j.jeee.20170504.11

Received: April 29, 2017; Accepted: May 9, 2017; Published: July 4, 2017

\begin{abstract}
Multi-antenna transmission already plays an important role in current generations of mobile communication and will be even more central in the $5 \mathrm{G}$, due to the physical limitations of small antennas. Path loss between a transmitter and receiver does not change as a function of frequency, as long as the effective aperture of the transmitting and receiving antennas does not change. The antenna aperture does reduce in proportion to the square of the frequency, and that reduction can be compensated by the use of higher antenna directivity. The $5 \mathrm{G}$ radio will employ hundreds of antenna elements to increase antenna aperture beyond what may be possible with current cellular technology. This paper presents a new compact broadband planar slot antenna for such kind of wireless communication applications. To develop this structure we have conducted a design based on on the microstrip line combined with a slot technique and a modified geometry antenna in order to enlarge the bandwidth and adapting the impedance thus minimizing distortion in order to avoid high crosstalk and radiation. The proposed antennas have been successfully designed, optimized, miniaturized and simulated by using Momentum software integrated into ADS "Advanced Design System" and CADFEKO. The final broadband antennas are operating in 9.84GHz on ADS and 9.5 GHz on FEKO respectively with a return loss less than $-10 \mathrm{~dB}$.
\end{abstract}

Keywords: Microstrip Antennas, Rectangular Patch, Millimeter Wave, Slot Antenna

\section{Introduction}

Over the years, interest in mobile data have and is still experiencing an unprecedented growth. This growth is further fuelled by the emergence of new data hungry devices like e-book readers, notebooks, smartphones, tablets etc. It is envisaged that the next generation of wireless system informally called the fifth Generation $(5 \mathrm{G})$ will be data intensive and will support myriad of connectivity between machines, humans, devices and a lot more. In short, there will be connectivity between anything, anywhere and anytime. Hence the need for available spectrum to support these services becomes critical. The sub $3 \mathrm{GHz}$ spectrum has since being exhausted while the $3-300 \mathrm{GHz}$ remains underutilized. In the $3-300 \mathrm{GHz}$ span, $3-30 \mathrm{GHz}$ is called Super High Frequency (SHF) band while $30-300 \mathrm{GHz}$ is known as Extremely High Frequency (EHF) or millimeter wave band [1]. But according to Zhouyue and Khan [2], since they share the same propagation characteristics they can be referred collectively as millimeter wave band with wavelength ranging between 1 to $100 \mathrm{~mm}$.

This requirement raises numerous design challenges to achieve a reasonable trade-off between technological design issues and commercial criteria - low cost, small size, radiation efficiency, antenna gain, broadband performance, and so on mainly at millimetric wave bands $[3,4]$.

Microstrip antennas are widely used in these applications due to their attractive features such as low profile, broadband, small in size, light in weight, low cost and ease of fabrication $[5,6]$. In addition to this, they are extremely compatible to other radio frequency microwave integrated circuit in manufacturing and low coupling affect in installation $[7,8]$.

Combination of the microstrip line, antenna geometry, and a variety of slot shapes is a solution to improve, enlarge the antenna operating bandwidth and adapting the input impedance $[9,10]$. In this paper, a new low cost broadband microstrip antenna is designed by using slot techniques and it 
is simulated by using FEKO Simulator and ADS to obtain the expected frequency band with suitable technical specifications.

\section{Antenna Structure Design}

\subsection{Design Procedures}

The design of rectangular patch antenna is based on the procedure given by [Luxey][11]. This can be used for a first sizing. The optimization can then be carried out using an electromagnetic simulator.

We consider a ground plane of perfect and infinite we have the following equations:

$$
W=\frac{\delta}{2} \sqrt{\frac{2}{1+\varepsilon_{r}}}
$$

With:

$\delta=\frac{c}{f_{r}}$

$c:$ Speed of light

$f_{r}$ : Resonant frequency

$W$ : Patch Width

$\mathcal{E}_{r}:$ Relative permittivity

$$
h \leq \frac{0.3 * c}{2 \pi * f_{r} * \sqrt{\varepsilon_{r}+1}}
$$

With:

$h$ : Maximum height

$$
\Delta L=h * 0.412 * \frac{\left(\varepsilon_{r e f}+0.3\right) *\left(\frac{W}{h}+0.264\right)}{\left(\varepsilon_{r e f}-0.258\right) *\left(\frac{W}{h}+0.8\right)}
$$

With:

$\Delta L:$ Extending the Patch Length

$$
\begin{gathered}
\mathcal{E}_{r e f}=\frac{\varepsilon_{r}+1}{2}+\frac{\varepsilon_{r}-1}{2} \cdot\left[1+12 \cdot \frac{h}{W}\right]^{-\frac{1}{2}} \\
L_{e f f}=\frac{\delta}{2 \sqrt{\varepsilon_{e f f}}}=\frac{c}{2 \cdot f_{r} \cdot \sqrt{\varepsilon_{e f f}}}
\end{gathered}
$$

With:

$L_{\text {eff }}$ : Effective patch length

$\varepsilon_{\text {ref }}:$ Reference permittivity

$\varepsilon_{\text {eff }}$ : Effective permittivity

$$
L=L_{\text {eff }}-2 \Delta L
$$

With:

$L:$ Physical Patch Length

$$
W_{g}=W+\frac{c}{20 \cdot f_{r}} L_{g}=L+\frac{c}{20 \cdot f_{r}},
$$

With:

$L_{g}$ : Ground length

$W_{g}$ : Ground width

The position of the power is given by the equation (8)

$$
Y_{F}=\frac{W}{2} X_{F}=\frac{L}{2 \sqrt{\varepsilon_{r e f}}},
$$

The geometry of the proposed broadband antenna slot structure is shown in "Figure 1". It is implemented on a low cost FR4 epoxy substrate with an area of $4 \times 26 \mathrm{~mm}^{2}$, a dielectric constant $\varepsilon_{r}=4.4$, a thickness $\mathrm{h}=1.6 \mathrm{~mm}$. The antenna is is excited with $50 \Omega$ characteristic impedance.

The design is based on a microstrip line, with the use of slot techniques, taken into consideration the gain and directivity. The purpose of the slot was to control the radiation pattern in order to obtain an increased bandwidth.

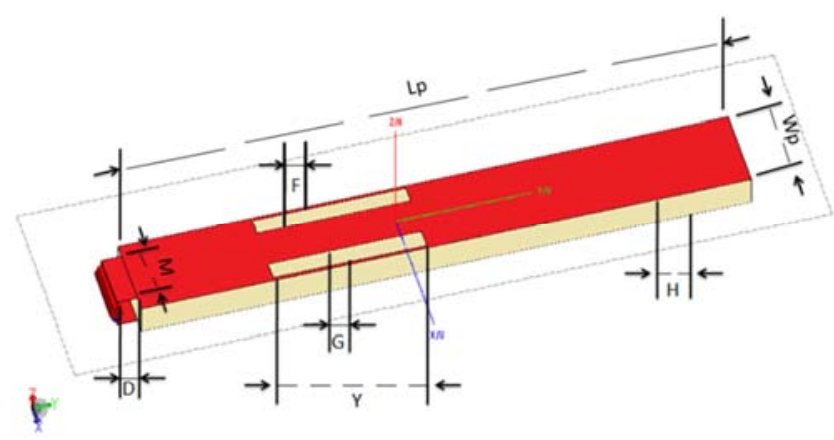

Figure 1. The Geometry of the proposed antenna.

\subsection{Simulation and Comparison}

The optimization of theoretical parameters design is done by using Momentum electromagnetic software integrated into ADS, which contains different techniques and calculation methods [12]. For comparison, we have conducted another study by using FEKO. After many optimizations and miniaturizations, the dimensions of the final optimized design structure are listed in Table 1.

Table 1. Antenna dimensions in (mm).

\begin{tabular}{ll}
\hline Variables & Value \\
\hline Lp & 26 \\
Wp & 4 \\
G & 0.85 \\
H & 1.6 \\
Y & 7.5 \\
M & 2.7 \\
D & 2 \\
F & 0.89 \\
\hline
\end{tabular}


To obtain these final dimensions we have conducted different simulations using the optimization and miniaturization techniques integrated into ADS are presented in "Figure 2" showing the return loss and bandwidth improvement for successive slots geometry. The final circuit is operating in a large frequency band between $6.37 \mathrm{GHz}$ and $14.06 \mathrm{GHz}$.
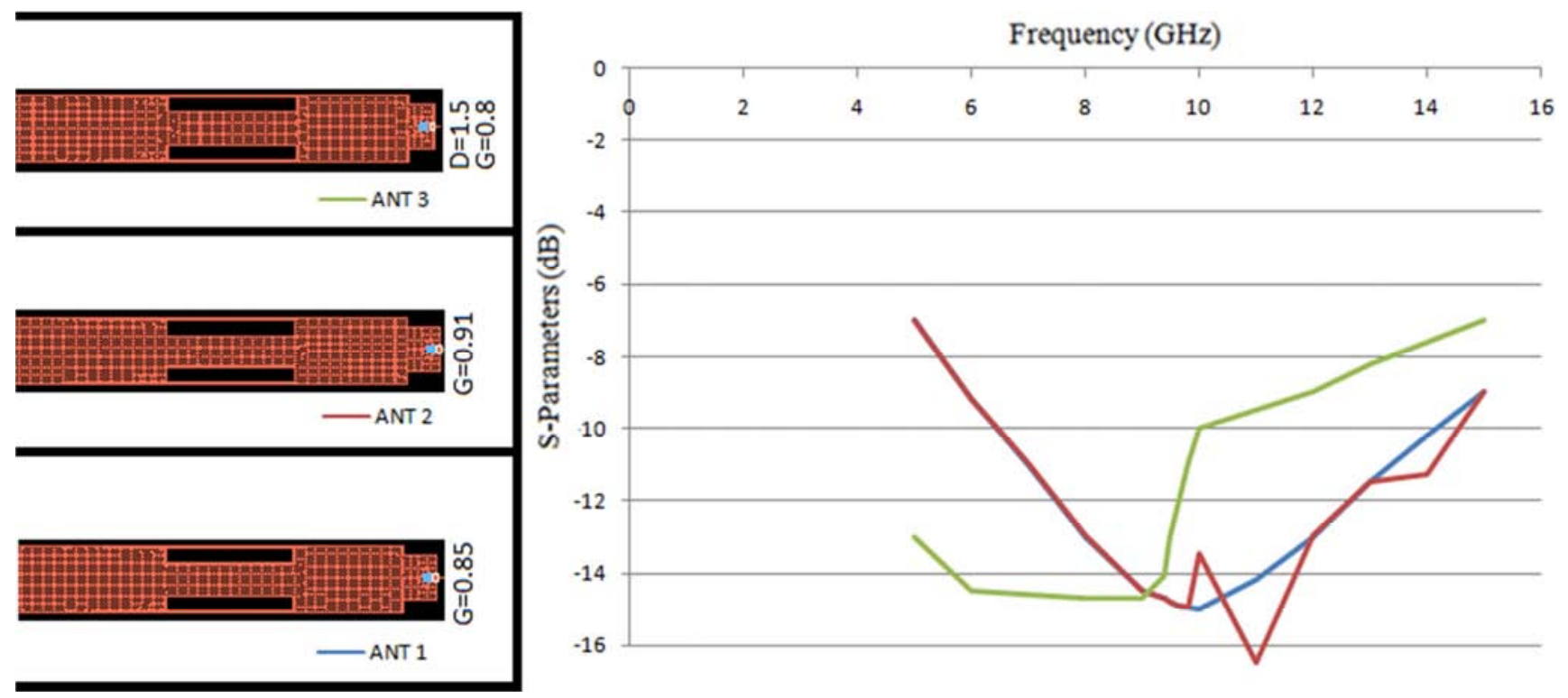

Figure 2. Slot dimensions in (mm) and The Return loss Vs frequency.

As shown in "Figure 3" the antenna validated into ADS simulation has a bandwidth from $6.37 \mathrm{GHz}$ to $10.06 \mathrm{GHz}$ and $f_{r}$ $=9.84 \mathrm{GHz}$. for the comparison of these results we kept the same antenna geometry and we have conducted another simulation by FEKO, we found that the bandwidth is from $9.38 \mathrm{GHz}$ to $9.63 \mathrm{GHz}$ and $f_{r}=9.5 \mathrm{GHz}$.

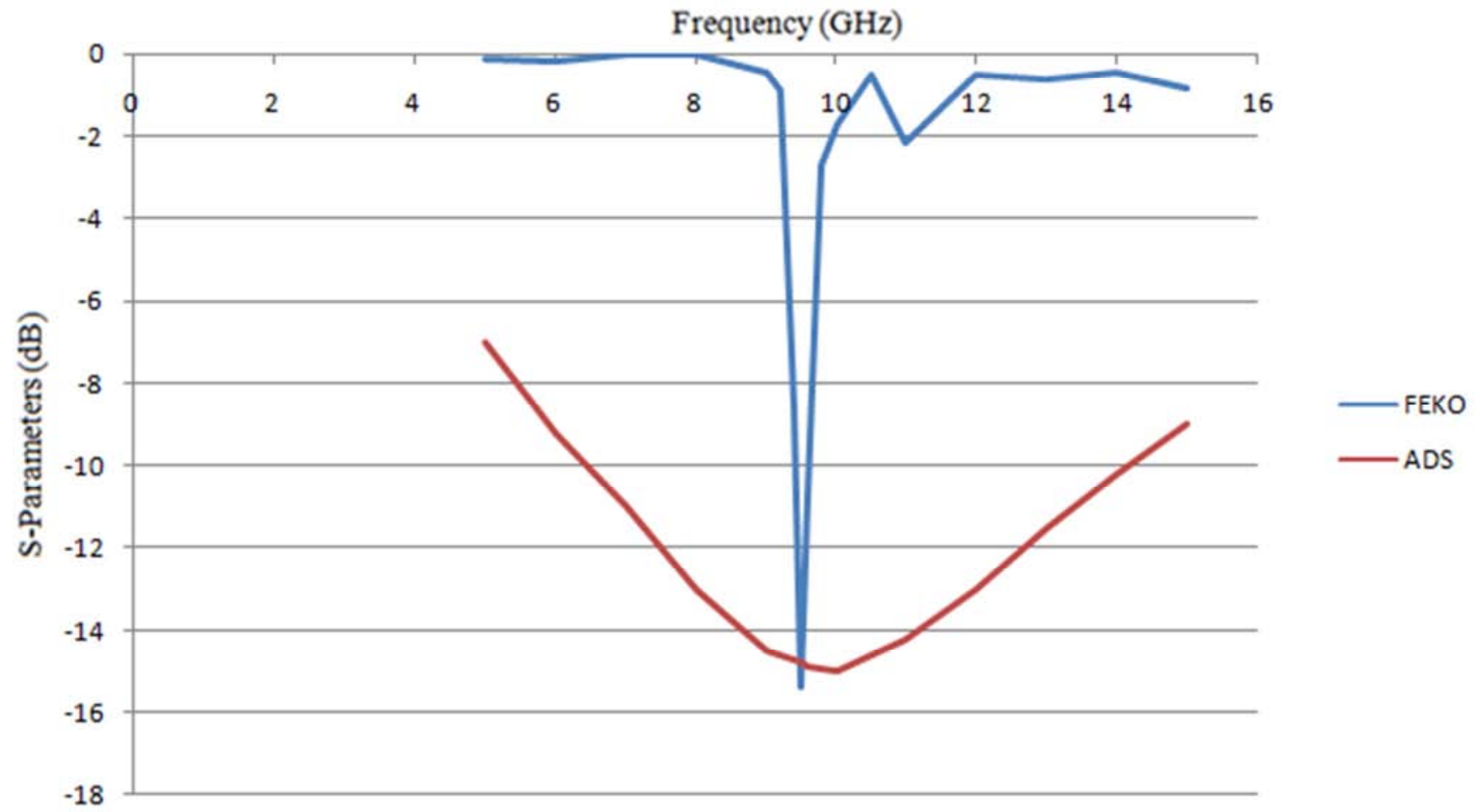

Figure 3. The return loss versus frequency on ADS and FEKO.

We have studied the behavior of the phase of reflection coefficient S11 versus frequency and it is presented in "Figure 4". It can be noticed that the phase seems to be linear across the UB frequency range. 




Figure 4. The phase versus frequency in $\mathrm{GHz}$.

For radiation pattern, "Figure 5" presents the ADS 3D antenna radiation for $9.84 \mathrm{GHz}$; it is omnidirectional antenna which radiates radio wave power uniformly in all directions in one plane.

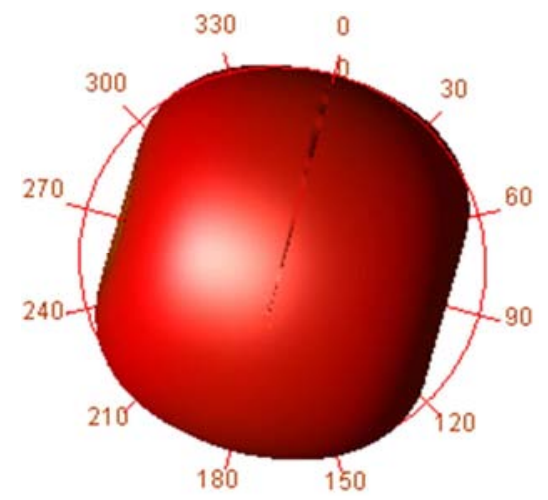

Figure 5. The 3D radiation pattern of the antenna optimized on ADS.

For a comparison and validation radiation pattern of the antenna at $9.5 \mathrm{GH}$ is simulated using FEKO and it is illustrated in "Figure 6".

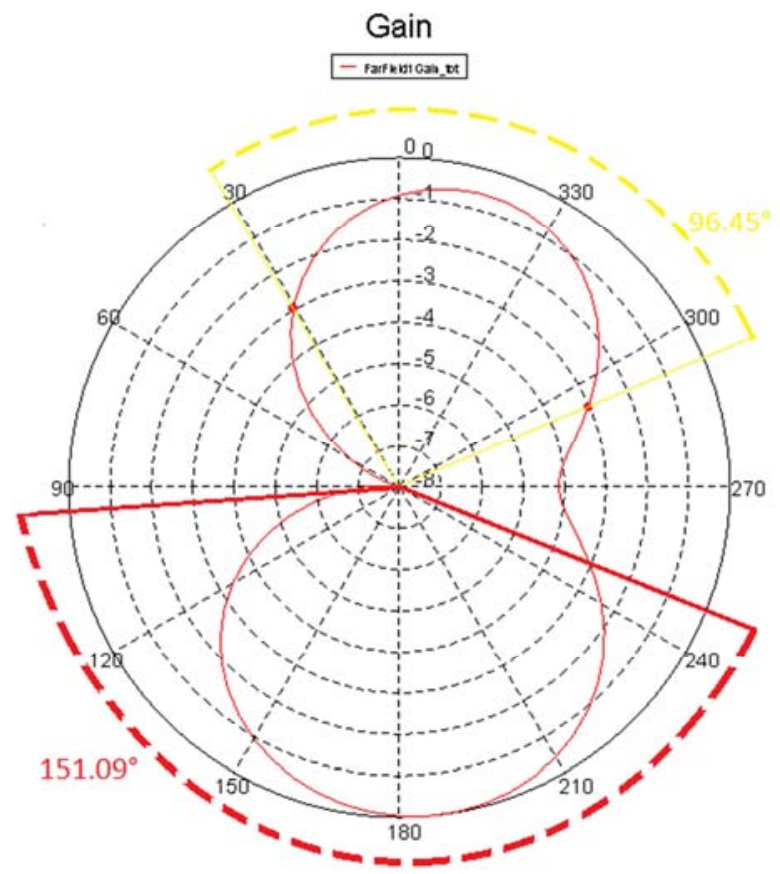

Figure 6. The Radiation pattern at $9.5 \mathrm{GHz}$ on FEKO.
According to the representation of the radiation pattern, the angle of opening of this antenna is $151,09^{\circ}$, which is beneficial for a base station for maximum coverage during the mobility of the subscriber. In our case, we took the rear lobe as a reference which means that the rear / front ratio is $11.13 \mathrm{~dB}$ therefore good power transmission.

Based on the simulations results presented above and for same antenna geometry, the results obtained are nearly the same in terms of resonate frequency using ADS and FEKO with acceptable and reasonable bandwidth 250MHZ.

\section{Conclusion}

In this study, the conception and the simulation of a new low cost rectangular planar antenna is performed based on the theoretical equations to find out the initial antenna dimensions. in other hand after studying several researches, the elaborated design is based on different methods such as a microstrip line combined with a slot technique and a modified geometry antenna in order to enlarge the bandwidth and adapting the impedance. Two high electromagnetic simulators FEKO and ADS are used to obtain the suitable 5G Antenna Geometry operating in $9.5 \mathrm{GHz}$ with a return loss less than $-10 \mathrm{~dB}$. The simulation results obtained by the two simulators are in agreement which validate the proposed antenna structure.

\section{References}

[1] Niu, Y., Li, Y., Jin, D. et al. Wireless Networks. November 2015, Volume 21, Issue 8, pp 2657-2676.

[2] Z. Pi and F. Khan. 2011. An introduction to millimeter-wave mobile broadband systems. Communications Magazine, IEEE. 49: 101-107.

[3] W. Roh et al., "Millimeter-Wave Beamforming as an enabling technology for 5G cellular communications: theoretical feasibility and prototype results", IEEE Comm. Magazine, pp. 106-113, Feb. 2014.

[4] E. Levine, G. Malamud, S. Shtrikman, D. Treves, "A study of microstrip array antennas with the feed network", IEEE Transactions on Antennas and Propagation, vol. 37, pp. 426-434, 1989.

[5] M. A. Saed "Reconfigurable Broadband Microstrip Antenna Fed by A Coplanar Waveguide", PIER 55, pp 227-239, 2005.

[6] W. Q. Cao, B. N. Zhang, A. J. Liu, T. B. Yu, D. S. Guo, and Y. Wei, "Gain Enhancement for Broadband Periodic Endfire Antenna by Us ing Split -ring Resonator Structures," IEEE Trans. Antennas Propag., vol. 60, no 7, pp. 3513-3516, 2012.

[7] M. Bialkowski, and A. Abbosh, "Design of UWB planar antenna with improved cut-off at the out-of-band frequencies," IEEE Antennas Wireless Prop Lett, vol. 7, pp. 408-410, 2008

[8] C. H. Chen, E. K. N. Yung, and B. J. Hu, "Miniaturized CPW-fed circularly polarized corrugated slot antenna with meander line loaded,'Electron. Lett., vol. 43, no. 25, pp. 14041405, Dec. 6, 2007. 
[9] G. DeJean, R. L. Li, J. Laskar, and M. M. Tentzeris "Circularly Polarized Loop Antennas with a Parasitic Element for Bandwidth Enhancement”, pp. 401 - 404 vol, 1B, 2005.

[10] M. Sumi, K. Hirasawa, and S. Shi, “Two rectangular loops fed in series for broadband circular polarization and impedance matching”, IEEE Trans. Antennas Propagation, vol. 52, no. 2, pp. 551-554, Feb. 2004.
[11] P. Ciais, R. Staraj, G. Kossiavas, C. Luxey, «Design of an Internal Quad-Band Antenna for Mobile Phones», IEEE Microwave and Wireless Components Letters, vol. 14, no 4, p. 148-150, April. 2004.

[12] Advanced Design System http://www.home.agilent.com/agilent/home.jsp
(ADS) 